\title{
Protocol
}

\section{Comparing skill enhanced and standard cognitive behavioral therapy for depression: a protocol for a randomized trial}

\author{
Samuel T. Murphy, Brooklynn Bailey, Graham C. Bartels, Lisa N. Vittorio, \\ Megan L. Whelen, Robert J. Zhou, Daniel R. Strunk*
}

Department of Psychology, The Ohio State University, Columbus, Ohio, United States of America

Received: 24 June 2021

Revised: 13 September 2021

Accepted: 20 September 2021

\section{*Correspondence:}

Daniel R. Strunk,

E-mail: strunk.20@osu.edu

Copyright: (c) the author(s), publisher and licensee Medip Academy. This is an open-access article distributed under the terms of the Creative Commons Attribution Non-Commercial License, which permits unrestricted non-commercial use, distribution, and reproduction in any medium, provided the original work is properly cited.

\begin{abstract}
Background: Cognitive behavioral therapy (CBT) features prominently among the first-line treatments for depression. The development of CBT skills is associated with both symptom reductions and reduced risk for relapse, suggesting that improvements in CBT skills might be important to the benefits of CBT. Nonetheless, standard CBT places modest emphasis on the development of these skills.

Methods: In this paper, we describe a randomized clinical trial testing the benefits of a variation of CBT that places greater emphasis on teaching patients CBT skills and makes patients' independent use of these skills a central therapeutic goal. Patients in this study ( 75 per condition) are being randomized to 12 weeks of standard or skill enhanced CBT for depression. The primary outcomes are change in symptoms and change in CBT skills. We will also evaluate the process of change in the treatments.

Conclusions: This study has the potential to suggest an approach to enhancing CBT outcomes through greater focus on patients developing and independently using CBT skills. It will also provide an important context for understanding the role that CBT skills play in the therapeutic outcomes of CBT.

Trial registration: The trial has been registered in ClinicalTrials.gov. Registration number: NCT04673513; registered on 17 December 2020.
\end{abstract}

Keywords: Cognitive behavioral therapy, CBT skills, Emotion regulation, Depression, Protocol

\section{INTRODUCTION}

Cognitive behavioral therapy (CBT) is the most wellstudied of all psychotherapies for depression, with evidence from clinical trials showing that it is as effective as pharmacotherapy as an acute treatment, with more enduring effects. ${ }^{1}$ However, even the best available treatments for depression result in inadequate symptom reduction for a substantial portion of clients. ${ }^{2}$ Furthermore, the portion of patients who respond to CBT and maintain their gains is still relatively low, with only a minority of patients exhibiting sustained responses. ${ }^{3,4}$ Thus, there is need to find ways to enhance the outcomes achieved with depression treatments.
One important approach to enhancing CBT is to develop interventions that further leverage what patients learn in treatment. $^{5}$ A number of studies suggest that patients learn to use CBT skills. Therapists' use of procedures intended to teach cognitive behavioral skills predict patients' use of these skills, and patients' development and use of these skills in turn predict symptom improvements. ${ }^{6-8}$ Among patients who have responded to CBT, their mastery and independent use of CBT skills predicts lower risk of relapse. ${ }^{9}$ Nonetheless, because this evidence is naturalistic, the findings do not establish whether interventions intended to foster skills causally contribute to positive outcomes. Only studies that involve 
randomization to different treatment procedures can resolve this issue.

In a focused experimental comparison, Bruijniks and colleagues found that a brief written intervention promoting CBT skills resulted in greater skill on a subsequent test than a no intervention comparison. ${ }^{10}$ Although there were no differences in mood or depressive symptoms, this finding was the first experimental evidence that intervention procedures can foster CBT skills. In a subsequent study, Bruijniks and colleagues compared a 90-minute CBT skills intervention to a control condition involving exposure to theories of automatic thinking. ${ }^{11}$ The skill condition resulted in more CBT skills following a subsequent mood induction, though there were no differences between conditions in reductions in dysfunctional beliefs or emotions. Findings from these studies provide some evidence that skill promoting interventions impact skill development. However, more extensive training or the sustained use of skills may be required to achieve positive therapeutic outcomes.

In this study, we compare standard CBT to CBT: skillenhanced (CBT-SE), a version of CBT that makes patients' development and independent use of these skills a central therapeutic goal. We detail the protocol for an ongoing trial making this comparison. The primary aims of the study are to compare standard CBT to CBT-SE on therapeutic outcomes, including symptom change, change in functioning, change in CBT skills, and risk of relapse following treatment.

\section{METHODS}

\section{Participants}

Study participants will be adults aged 18 or older currently living in Ohio with stable access to the internet. Inclusion criteria for this study are: diagnosis of major depressive disorder (MDD); access to a reliable internet connection (to participate in virtual sessions); and being able and willing to give informed consent. Exclusion criteria are: current or past diagnosis of bipolar disorder or a psychotic disorder; moderate or severe substance use in the past 6 months (where "moderate" is defined as 4-5 symptoms and "severe" is defined as 6 or more symptoms of those listed in DSM-5 for each of the substance-related disorders); current disorder other than MDD if it constitutes the predominant aspect of the clinical presentation and if it requires treatment other than that being offered; current participation in a treatment for an emotional disorder, including any individual psychotherapy or psychiatric medications used to treat a mood or anxiety disorder; clear indication of secondary gain (e.g. court-ordered treatment); and current suicide risk or significant intentional self-harm in the last six months sufficient to preclude treatment on an outpatient basis.
All diagnoses will be assessed with the structured clinical interview for the diagnostic and statistical manual of mental disorders-5 (SCID-5). ${ }^{12}$

\section{Recruitment}

We anticipate a final sample of 150 participants $(75$ per condition). We began enrolling participants in December 2020 and anticipate enrolling participants over a period of three years. To recruit participants, we are using advertisements on social media websites, postings on websites frequented by those looking for treatment opportunities (e.g. https://clinicaltrials.gov), media releases, and referrals from other clinics.

\section{Procedure}

This study is a randomized clinical trial. Eligible participants are randomized to standard CBT or CBT: skill-enhanced (CBT-SE). Treatment is provided over 12 weeks. Participants can utilize two booster sessions over a six month follow-up period following treatment.

Potential participants are first directed to a study website. On this page, they can either express interest in being contacted by for a pre-screening interview via phone or complete an initial prescreening questionnaire online (with phone follow-up as needed). If a participant is potentially eligible following the pre-screening, they are offered the opportunity to schedule an intake appointment. Prior to that appointment, they are sent a consent form and link to online questionnaires. They respond to the online questionnaires prior to the intake. At the intake appointment, participants are provided detailed information about the study and are offered the opportunity to consent to participate further. The intake assessment includes interview assessments of key variables, including CBT skills. Following this consent, eligibility is determined and those who are eligible are randomized to a treatment condition. The randomization sequence for the trial was generated using the maximal procedure ( $\mathrm{r}$ package MP boost) with a maximum tolerated imbalance of four. ${ }^{13}$ A third party sealed each assignment in an envelope. After a participant is deemed eligible, the study principal investigator (DRS) unseals the requisite envelope. ${ }^{14}$ Participants are then quasirandomized to a study therapist, with chance of randomization to each therapist determined by the number of openings in each therapist's caseload as well as the proportion of CBT and CBT-SE cases the therapist has been assigned.

Clients and therapists providing treatment are asked to respond to surveys prior to and following each session. During week four of treatment, clients complete an additional assessment. This assessment includes a selfreport survey packet evaluating symptom severity, CBT skills, and vulnerability factors. An interview assessment also evaluates CBT skills and symptom severity. A 
similar assessment evaluating these same variables occurs at the end of treatment, during week 12. Finally, participants are asked to complete follow up surveys each month for six months following treatment, with surveys assessing symptom severity, CBT skills, and the use of any mental health treatments outside the study protocol.

\section{Incentives}

Participants are offered incentives to complete most of the study assessments in the form of gift cards. An incentive of $\$ 2$ per session for up to $\$ 32$ total for responding to pre- and post-session questionnaires is being offered. There is an additional $\$ 15$ incentive for the post-treatment assessment. For the monthly follow-up assessments occurring for six months following treatment, an incentive of $\$ 5$ per occasion is offered for a maximum of $\$ 30$. The maximum incentive per participant is $\$ 77$. In addition, two lottery prizes of $\$ 100$ gift cards are also being offered. For the first, participants earn a chance in the drawing for each session survey they submit. For the second, participants earn a chance in the drawing for each monthly follow-up survey they submit.

\section{Treatments}

Both treatments provided as part of the study are cognitive behavioral therapies for depression. Standard CBT is being provided as described in the original treatment manual, with additional procedures as recommended in a more recent text. ${ }^{15,16}$ Standard CBT is based on a model emphasizing the importance of identifying and correcting overly negative views that serve to maintain depression. CBT is an active and structured treatment. Therapists work collaboratively with patients, individualizing therapy targets for each patient. Patients learn to identify their automatic thoughts and evaluate their accuracy, revising their views as needed. This evaluation includes considering evidence, alternative perspectives, and the realistic consequences if their thoughts were true. CBT includes both cognitive and behavioral strategies. Behavioral strategies include self-monitoring of moods and activities, scheduling activities that may promote a sense of pleasure or accomplishment, and structuring activities to break down a task into more manageable steps. Assertiveness training and role playing can also be used. Whenever possible, behavioral strategies are used in the service of promoting cognitive change.

As treatment progresses, a focus is placed on not only identifying automatic thoughts, but on identifying and modifying underlying assumptions and core beliefs. Behavioral experiments testing a patient's core beliefs are an important part of efforts to bring about more fundamental cognitive change. Patients also learn to use the skills of CBT increasingly independently as treatment progresses.
CBT-SE includes and builds upon the core principles and procedures of standard CBT with an increased focus on CBT skill development. ${ }^{16,17}$ The added focus on skills begins in session one with a treatment rationale that places greater emphasis on the importance of CBT skill developments as a central goal of treatment. Throughout treatment, therapists continue to emphasize the importance of CBT skill development and highlight how CBT skills can be used to combat depressive symptoms and achieve goals. Additional phone coaching is used to reinforce and help patients effectively utilize CBT skills between sessions. Throughout treatment, therapists work to help clients take greater and greater leadership as part of the therapeutic team. Therapists work to help clients identify take home points consistent with promoting skill use and development in each session. To further promote client mastery of skills, therapists offer clients brief supplementary videos that reinforce key CBT skills that they can review between sessions. Therapists also place greater emphasis on practicing skills through homework assignments, including a concerted focus on how skills can be used flexibly to overcome potential obstacles. Homework assignments in CBT-SE are also focused more strongly on promoting skill development and clients making a regular habit of using skills independently. Following sessions 4 and 10, skills assessments are performed by a clinician other than the therapist. The assessor provides feedback about the clients' competency in using CBT skills that serves to help the therapist and client focus their efforts more effectively. In later sessions, there is a considerable focus on anticipating and rehearsing the use of skills to cope with future stressors. Self-therapy sessions are introduced and practiced. These sessions involve clients independently doing the work that they might complete with a therapist in a regular session. By the end of treatment, the goal is for the clients to have a strong foundation in developing CBT skills and to have developed practices that will promote their maintenance and further development.

For both forms of CBT, the protocol involves twice weekly sessions for the first four weeks. From week 4 to week 8 , the therapist and client collaboratively decide whether to maintain twice weekly sessions or switch to once weekly sessions. Weekly sessions are provided from week 8 to week 12 . Therefore, across the 12 weeks, the protocol calls for 16 to 20 sessions. In both conditions, clients have the opportunity to access up to two booster sessions over the six month follow-up period.

\section{Materials}

\section{Diagnostic measure}

The SCID-5, research version for the diagnostic and statistical manual of mental disorders- 5 is used to assess whether participants meet formal criteria for specific disorders. ${ }^{12,17}$ At intake, the SCID-5 is administered by study personnel trained in its use. 


\section{Primary outcome measures}

CBT skills interview: This interview assesses client skills in using coping strategies taught in CBT. The measure was adapted from another interview-based CBT skills measure we utilized in a prior study, the competencies of cognitive therapy scale-interview (CCTS-I). ${ }^{18}$

Cognitive change, sustained version (CCS): The CCS is a 9-item self-report measure that assesses the extent to which participants experienced cognitive changes and used CBT skills since their previous therapy session. ${ }^{7}$

Quick inventory of depressive symptomatology-selfreport (QIDS-SR: The QIDS is a 16-item measure of depressive symptom severity. For this measure, participants report the frequency and severity of depressive symptoms in the past week. ${ }^{19}$

Ways of responding scale (WOR): The WOR is a measure of CBT skills. Participants respond with what their thoughts and actions would be for 6 hypothetical stressful situations, and trained coders evaluate their responses. ${ }^{20}$

\section{Secondary outcomes measures}

Quick inventory of depressive symptomatology-clinician version $(Q I D S-C)$ : The QIDS is a 16-item measure of depressive symptom severity. For the QIDS-C, an evaluator rates the items based on an interview with the respondent. ${ }^{19}$

Cognitive change, immediate version (CCI): The CCI is a 5 item self-report measure of the extent to which the client experienced cognitive change and utilized CBT skills during therapy sessions. This measure is given at the end of each session. ${ }^{7}$

Work social adjustment scale (WSAS): The WSAS is a 6item self-report measure of functioning. Items assess the extent to which the client's functioning is impaired with regard to the client's ability to work, manage problems at home, participate in social and private leisure activities, and form close relationships. ${ }^{21}$

Competencies of cognitive therapy scale - client and therapist (CCTS-SR and CCTS-TR): The CCTS-SR and CCTS-TR measure clients' mastery of cognitive and behavioral CBT skills. Both a therapist and client version are being used. The client version (i.e. CCTS-SR) is 29items. The therapist version (i.e. CCTS-TR) is 9 items. ${ }^{22}$

\section{Other outcome measures}

Patient health questionnaire (PHQ-9): The PHQ-9 is a self-report measure based on the diagnostic criteria for major depression. It has been used in primary care and research settings to assess depression severity, symptomatology, and functional impairment. A modified version of the PHQ-9 (i.e. we changed the wording in the instructions from "over the past 2 weeks" to "during the worst 2 weeks") is administered every month during the post-treatment follow-up period. Additional items were added to assess for any mental health treatment services the client may have sought. ${ }^{23}$

\section{Additional measures}

Alliance is being measured with client and therapist versions of the working alliance inventory-short form (WAI). ${ }^{24}$ The homework assessment client and therapist (HWA-C and HWA-T) is being used to measure homework assignment and engagement with these assignments. We also assess participants' memory of important points, principles, and experiences from the previous session using a single open-ended question adapted from the memory assessment used by Harvey and colleagues. ${ }^{25}$ The positive and negative affect schedule (PANAS) is being used as a measure of positive and negative mood. ${ }^{26}$ Anxiety symptoms are assessed using the generalized anxiety disorder questionnaire-7 (GAD-7). ${ }^{27}$ Hopelessness is assessed using the Beck hopelessness scale (BHS). ${ }^{28}$ Maladaptive thinking is assessed using the dysfunctional attitude scale (DAS). ${ }^{29}$ Rumination is assessed using the ruminative response scale (RRS). ${ }^{30,31}$ Maladaptive personality traits are assessed with the personality inventory for DSM-5 - brief form (PID-5-BF). ${ }^{32}$ Big Five personality traits are measured using the NEO personality inventory (NEOFFI). ${ }^{33}$ The proportionality of participants depressive responses is assessed using the proportionality of depressive reactions - self report (PDR). ${ }^{34}$ Stress is assessed using the perceived stress scale (PSS). ${ }^{35}$ Life events are assessed with the psychiatric epidemiology research interview life events rating scale (PERI). ${ }^{36}$ The interpersonal emotion regulation questionnaire (IERQ) is being used to measure respondents' interpersonal skills. ${ }^{37}$ the inventory of interpersonal problems-32 (IIP-32) is being used to assess interpersonal problems. ${ }^{38}$ Therapists and assessors report on predicted response patterns to depression treatment using the brief response profiles questionnaire (BRPQ), which is based on the work of DeRubeis and colleagues. ${ }^{39} \mathrm{We}$ are assessing whether specific events related to the novel coronavirus disease 2019 (COVID-19) have occurred in the client's life or in the lives of others they know. We are assessing clients' perceptions of the treatment using an adapted version of the credibility/expectancy questionnaire (CEQ). ${ }^{40} \mathrm{We}$ are assessing therapist adherence using a brief self-report measure after each session created for this study. Finally, we are using study specific materials to assess clients' treatment history using interview and self-report items.

\section{Analytic strategy}

We plan to test several outcomes for this study. We will assess treatment differences in change in CBT skills, cognitive change (across six measures), and depressive symptoms (on two measures) using hierarchical linear 
modeling. We also plan to evaluate change in functioning using a similar analytic approach. Finally, we plan to evaluate treatment differences in the risk of relapse over the course of the follow-up period using survival analysis.

\section{DISCUSSION}

This study will evaluate CBT-SE which was designed to leverage a promising putative mechanism of CBT: CBT skills. These skills are thought to be important to facilitating a positive response to acute treatment and may help to account for long-term benefits of CBT.7,22 The study involves a comparison of the benefits of CBTSE and standard CBT. We will compare both clinical outcomes as well as differences in skill acquisition. Although CBT skill acquisition has received considerable attention, this study will be the first to directly test whether an increased emphasis on skill development leads to superior improvements in CBT skills and clinical outcomes.

Tests using these measures will allow us to evaluate differences in skill acquisition over the course of treatment. We plan to test whether the greater focus on CBT skills translates into greater skill acquisition and positive therapeutic outcomes. We will also evaluate the process of change in both treatments. As we are comparing CBT-SE to standard CBT, a treatment known to be among the most efficacious treatments for depression, we will be able to determine if CBT-SE offers benefits that might surpass a current first-line treatment for depression. ${ }^{1}$

In recent years, there has been a renewed interest in efforts to personalize CBT based on moderators of intervention effects involve developing variations of CBT. One approach to researching these refinements has been to measure naturalistic variability in the use of therapeutic strategies and identify client characteristics that moderate the relation of different strategies with processes or treatment outcomes. ${ }^{41,42}$ In describing how such research could be used to inform treatment, Huibers and colleagues give a hypothetical example of a research finding that a focused cognitive intervention was particularly effective for women with high levels of anxiety and noted that this finding might lead researchers to propose using a personalized form of CBT using greater cognitive restructuring with these patients. ${ }^{43}$ This process involves a disconnect between the finding that informed the intervention and the personalized treatment. When researchers find moderation in these studies, the findings are taken to suggest that therapists should use an intervention approach more with clients who appeared to benefit more strongly from it. However, because clients were not randomized to the different treatment strategies, inferences about the benefits of each approach are much more tenuous. In addition, an assumption is made that therapists can utilize an intervention more fully to facilitate more positive therapeutic outcomes.
An alternative is to test treatments that vary in specific ways and then evaluate moderators in this context. An advantage of the latter approach is that it makes it clear what intervention strategies are indicated for patients with different characteristics. Studies that involve randomizing clients to different treatment approaches allow for more compelling tests of what therapy procedures work best for whom. ${ }^{44}$ Furthermore, because such comparisons involve manuals for each condition, it is clear how the findings can be used to inform clinical practice. Therapists would simply use the manual for the treatment that was found to be most beneficial for clients with certain characteristics.

\section{CONCLUSION}

In conclusion, empirically informed treatment refinements have the potential to meaningfully advance the effort to improve psychotherapy outcomes in the treatment of depression. This currently ongoing study could offer a means of enhancing the benefits of CBT for depression. If the CBT-SE condition is found to offer benefits beyond that of standard CBT, overall or for specific subsets of patients, this would indicate that outcomes in CBT could be enhanced by therapists working to enhance CBT skill development either generally or for those specific patients who would benefit more from this approach.

\section{Funding: No funding sources}

Conflict of interest: None declared

Ethical approval: The study was approved by the Institutional Ethics Committee

\section{REFERENCES}

1. Strunk DR, Adler AD, Hollon SD. Cognitive therapy of depression. DeRubeis RJ, Strunk DR, Editors. The Oxford handbook of mood disorders. Oxford University. 2017;411-22.

2. Cuijpers P, Karyotaki E, Weitz E, Andersson G, Hollon SD, van Straten A. The effects of psychotherapies for major depression in adults on remission, recovery and improvement: A metaanalysis. J Affect Disord. 2014;159:118-26

3. Cuijpers P, Hollon SD, van Straten A, Bockting C, Berking M, Andersson G. Does cognitive behaviour therapy have an enduring effect that is superior to keeping patients on continuation pharmacotherapy? A meta-analysis. BMJ Open. 2013;3.

4. Hollon SD, DeRubeis RJ, Shelton RC, Amsterdam JD, Salomon RM, O'Reardon JP, et al. Prevention of relapse following cognitive therapy vs medications in moderate to severe depression. Arch Gen Psychiatry. 2005;62(4):417-22.

5. Bruijniks SJ, DeRubeis RJ, Hollon SD, Huibers MJ. The potential role of learning capacity in cognitive behavior therapy for depression: A systematic review of the evidence and future directions for 
improving therapeutic learning. Clin Psychol Sci. 2019;7:668-92.

6. Hundt NE, Mignogna J, Underhill C, Cully JA. The relationship between use of CBT skills and depression treatment outcome: a theoretical and methodological review of the literature. Behav Ther. 2013;44(1):12-26.

7. Schmidt ID, Pfeifer BJ, Strunk DR. Putting the "cognitive" back in cognitive therapy: Sustained cognitive change as a mediator of in-session insights and depressive symptom improvement. J Consult Clin Psychol. 2019;87:446-56.

8. Stone SJ, Strunk DR. Fostering Cognitive Change in Cognitive Therapy of Depression: An Investigation of Therapeutic Strategies. Cognitive Therap Res. 2020;44:21-7.

9. Strunk DR, DeRubeis RJ, Chiu AW, Alvarez J. Patients' competence in and performance of cognitive therapy skills: Relation to the reduction of relapse risk following treatment for depression. J Consult Clin Psychol. 2007;75:523-30.

10. Bruijniks SJ, Sijbrandij M, Schlinkert C, Huibers MJ. Isolating therapeutic procedures to investigate mechanisms of change in cognitive behavioral therapy for depression. J Exp Psychopathol. 2018;9:2043808718800893.

11. Bruijniks SJ, Los SA, Huibers MJ. Direct effects of cognitive therapy skill acquisition on cognitive therapy skill use, idiosyncratic dysfunctional beliefs and emotions in distressed individuals: An experimental study. J Behav Therap Exp Psychiatr. 2020;67:101460.

12. First MB, Williams JBW, Karg RS, Spitzer RL. Structured Clinical Interview for DSM-5 (research version). American Psychiatric Association. 2015.

13. Salama I, Ivanova A, Qaqish B. Efficient generation of constrained block allocation sequences. Stat Med. 2008;27:1421-8.

14. Moher D, Hopewell S, Schulz KF, Montori V, Gøtzsche PC, Devereaux PJ, et al. CONSORT 2010 explanation and elaboration: Updated guidelines for reporting parallel group randomised trials. Int $\mathrm{J}$ Surg. 2012;10:28-55.

15. Beck AT, Rush AJ, Shaw BF, Emery G. Cognitive therapy of depression. Guilford Publications. 1979.

16. Beck AT, Davis DD, Freeman A. Cognitive therapy of personality disorders. Guilford Publications. 2015.

17. American Psychiatric Association. Diagnostic and Statistical Manual of Mental Disorders (DSM-5®). 2013. Available at: https://www.psychiatry.org/ psychiatrists/practice/dsm. Accessed on 14 June 2021.

18. Murphy ST, Cooper AA, Hollars SN, Strunk, DR. Who benefits from a cognitive vs. behavioral approach to treating depression? A pilot study of prescriptive predictors. Behav Therap. 2021;52(6):1433-48.

19. Rush AJ, Trivedi MH, Ibrahim HM, Carmody TJ, Arnow B, Klein DN, et al. The 16-Item Quick
Inventory of Depressive Symptomatology (QIDS), clinician rating (QIDS-C), and self-report (QIDSSR): a psychometric evaluation in patients with chronic major depression. Biol Psychiatry. 2003;54(5):573-83.

20. Barber JP, DeRubeis RJ. The ways of responding: A scale to assess compensatory skills taught in cognitive therapy. Behavioral Assessment. 1992;14:93-115.

21. Mundt JC, Marks IM, Shear MK, Greist JH. The Work and Social Adjustment Scale: a simple measure of impairment in functioning. $\mathrm{Br} J$ Psychiatry. 2002;180:461-4.

22. Strunk DR, Hollars SN, Adler AD, Goldstein LA, Braun JD. Assessing patients' cognitive therapy skills: Initial evaluation of the competencies of cognitive therapy scale. Cognitiv Ther Res. 2014;38:559-69.

23. Kroenke K, Spitzer RL. The PHQ-9: A new depression diagnostic and severity measure. Psychiatr Ann. 2002;32:509515.

24. Hatcher RL, Gillaspy JA. Development and validation of a revised short version of the Working Alliance Inventory. Psychotherap Res. 2006;16:12-25

25. Harvey AG, Dong L, Lee JY, Gumport NB, Hollon $\mathrm{SD}$, Rabe-Hesketh $\mathrm{S}$, et al. Can integrating the Memory Support Intervention into cognitive therapy improve depression outcome? Study protocol for a randomized controlled trial. Trials. 2017;18(1):539.

26. Watson D, Clark LA, Tellegen A. Development and validation of brief measures of positive and negative affect: the PANAS scales. J Personal Social Psychol.1988;54:1063-70.

27. Spitzer RL, Kroenke K, Williams JB, Löwe, B. A brief measure for assessing generalized anxiety disorder: the GAD-7. Arch Int Med. 2006;166:1092-7.

28. Beck AT, Steer RA. Beck Hopelessness Scale manual. The Psychological Corporation. 1988.

29. Weissman AN. The Dysfunctional Attitude Scale: A validation study. Dissertation Abstracts International, 1979;40:1389-90.

30. Nolen-Hoeksema S, Morrow J. A prospective study of depression and posttraumatic stress symptoms after a natural disaster: The 1989 Loma Prieta earthquake. J Personal Soc Psychol. 1991;61:115-21.

31. Whitmer A, Gotlib, IH. Brooding and reflection reconsidered: A factor analytic examination of rumination in currently depressed, formerly depressed, and never depressed individuals. Cognitiv Therap Res. 2011;35:99-107.

32. Krueger RF, Derringer J, Markon KE, Watson D, Skodol AE. Initial construction of a maladaptive personality trait model and inventory for DSM-5. Psychol Med. 2012;42(9):1879-90.

33. Costa PT, McCrae RR. NEO PI-R professional manual. Psychological Assessment Resources, Inc. 1992. 
34. Pfeifer BJ, Strunk DR. Assessing the Disproportionality of Depressive Reactions to Life Stress. Clin Psychol Psychother. 2021.

35. Cohen S, Kamarck T, Mermelstein R. Perceived Stress Scale. Measuring Stress: A Guide for Health and Social Scientists. 1994;10:1-2.

36. Dohrenwend BS, Krasnoff L, Askenasy AR, Dohrenwend BP. Exemplification of a method for scaling life events: The PERI Life Events Scale. J Health Soc Behav. 1978;19:205-29.

37. Hofmann SG, Carpenter JK, Curtiss J. Interpersonal Emotion Regulation Questionnaire (IERQ): Scale Development and Psychometric Characteristics. Cognit Ther Res. 2016;40(3):341-56.

38. Barkham M, Hardy GE, Startup M. The IIP-32: A short version of the Inventory of Interpersonal Problems. Br J Clin Psychol. 1996;35:21-35.

39. DeRubeis RJ, Gelfand LA, German RE, Fournier JC, Forand NR. Understanding processes of change: How some patients reveal more than others-and some groups of therapists less - about what matters in psychotherapy. Psychother Res. 2014;24:419-28.

40. Devilly GJ, Borkovec TD. Psychometric properties of the credibility/expectancy questionnaire. J Behav Therap Exp Psychiatr. 2000;31:73-86.
41. Fitzpatrick OM, Whelen ML, Falkenström F, Strunk DR. Who benefits the most from cognitive change in cognitive therapy of depression? A study of interpersonal factors. J Consult Clin Psychol. 2020;88(2):128-36.

42. Sasso KE, Strunk DR, Braun JD, DeRubeis RJ, Brotman MA. Identifying moderators of the adherence-outcome relation in cognitive therapy for depression. J Consult Clin Psychol. 2015;83:976-84.

43. Huibers MJH, Lorenzo-Luaces L, Cuijpers P, Kazantzis N. On the Road to Personalized Psychotherapy: A Research Agenda Based on Cognitive Behavior Therapy for Depression. Front Psychiatry. 2021;11:607508.

44. Cohen ZD, DeRubeis RJ. Treatment selection in depression. Ann Rev Clin Psychol. 2018;14:209-36.

Cite this article as: Murphy ST, Bailey B, Bartels GC, Vittorio LN, Whelen ML, Zhou RJ, et al. Comparing skill enhanced and standard cognitive behavioral therapy for depression: a protocol for a randomized trial. Int J Clin Trials 2020;7(4):285-91. 